\title{
Acute Ischemic Stroke Involving Both Anterior and Posterior Circulation Treated by Endovascular Revascularization for Acute Basilar Artery Occlusion via Persistent Primitive Trigeminal Artery
}

\author{
Taichiro Imahori, M.D., Atsushi Fujita, M.D., Ph.D., Kohkichi Hosoda, M.D., Ph.D., Eiji Kohmura, M.D., Ph.D. \\ Department of Neurosurgery, Kobe University Graduate School of Medicine, Kobe, Japan
}

We report a case of acute ischemic stroke involving both the anterior and posterior circulation associated with a persistent primitive trigeminal artery (PPTA), treated by endovascular revascularization for acute basilar artery (BA) occlusion via the PPTA. An otherwise healthy 67-year-old man experienced sudden loss of consciousness and quadriplegia. Magnetic resonance imaging showed an extensive acute infarction in the right cerebral hemisphere, and magnetic resonance angiography showed occlusion of the right middle cerebral artery (MCA) and BA. Because the volume of infarction in the territory of the right MCA was extensive, we judged the use of intravenous tissue plasminogen activator to be contraindicated. Cerebral angiography revealed hypoplasia of both vertebral arteries and the presence of a PPTA from the right internal carotid artery. A microcatheter was introduced into the BA via the PPTA and revascularization was successfully performed using a Merci Retriever with adjuvant low-dose intraarterial urokinase. After treatment, his consciousness level and right motor weakness improved. Although persistent carotid-vertebrobasilar anastomoses such as a PPTA are relatively rare vascular anomalies, if the persistent primitive artery is present, it can be an access route for mechanical thrombectomy for acute ischemic stroke.

Key Words : Endovascular revascularization · Mechanical thrombectomy · Acute ischemic stroke · Basilar artery occlusion · Persistent primitive trigeminal artery $\cdot$ Persistent carotid-vertebrobasilar anastomosis.

\section{INTRODUCTION}

A persistent primitive trigeminal artery (PPTA) is a relatively rare vascular anomaly of persistent carotid-vertebrobasilar anastomoses $^{22)}$. Acute ischemic stroke associated with these cerebral vascular anomalies might have an unusual pattern of involved territories. To date, among the small number of reported cases of acute ischemic stroke associated with PPTA ${ }^{1-5,7,9,10,17,18,20,21,23)}$, most involved minor ischemic stroke; only a few cases of major stroke such as an acute basilar artery (BA) occlusion have been reported in the literature ${ }^{7)}$.

Simultaneous occurrence of acute major cerebral artery occlusions in both the anterior and posterior circulation is a rare life-threatening condition. Although these cases result in a fatal outcome if revascularization is not performed, most cases are not indicated for intravenous thrombolysis therapy because of the extensive infarctions. However, there might be an opportunity for treatment of the posterior circulation by endovascular revascularization, which can selectively restore the patency of an occluded target vessel, because the treatable time window for the posterior circulation is said to be longer than that of the anterior circulation.

We report a rare case of acute ischemic stroke involving both the anterior and posterior circulation associated with a PPTA, treated by endovascular revascularization of the posterior circulation. The present case is the first report of acute BA occlusion that was successfully treated by endovascular revascularization via the PPTA.

\section{CASE REPORT}

An otherwise healthy 67-year-old man suddenly lost con-

- Received : February 1, 2015 • Revised : April 29, 2015 • Accepted : May 20, 2015

- Address for reprints : Taichiro Imahori, M.D.

Department of Neurosurgery, Kobe University Graduate School of Medicine, 7-5-1 Kusunoki-cho, Chuo-ku, Kobe, Hyogo 650-0017, Japan

Tel : +81-78-382-5966, Fax : +81-78-382-5979, E-mail : taichiro.imahori@gmail.com

- This is an Open Access article distributed under the terms of the Creative Commons Attribution Non-Commercial License (http://creativecommons.org/licenses/by-nc/3.0) which permits unrestricted non-commercial use, distribution, and reproduction in any medium, provided the original work is properly cited. 


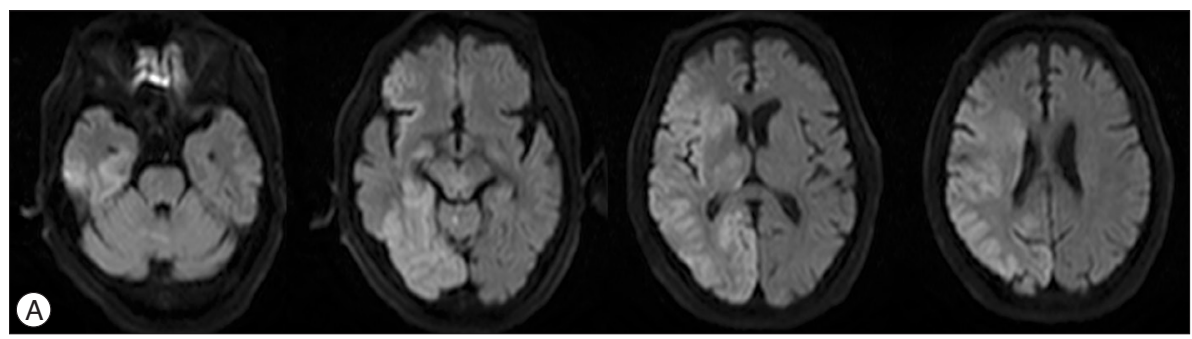

Fig. 1. A : Magnetic resonance diffusion-weighted imaging on admission showing an extensive acute infarction in the right cerebral hemisphere. B : Magnetic resonance angiography on admission showing occlusion of the right middle cerebral artery and basilar artery (BA). Bilateral extra-cranial vertebral arteries are comparatively well visualized, but bilateral intracranial VAs and the BA are poorly visualized. An unusual branch arising from the precavernous portion of the right internal carotid artery can be seen (white arrow).

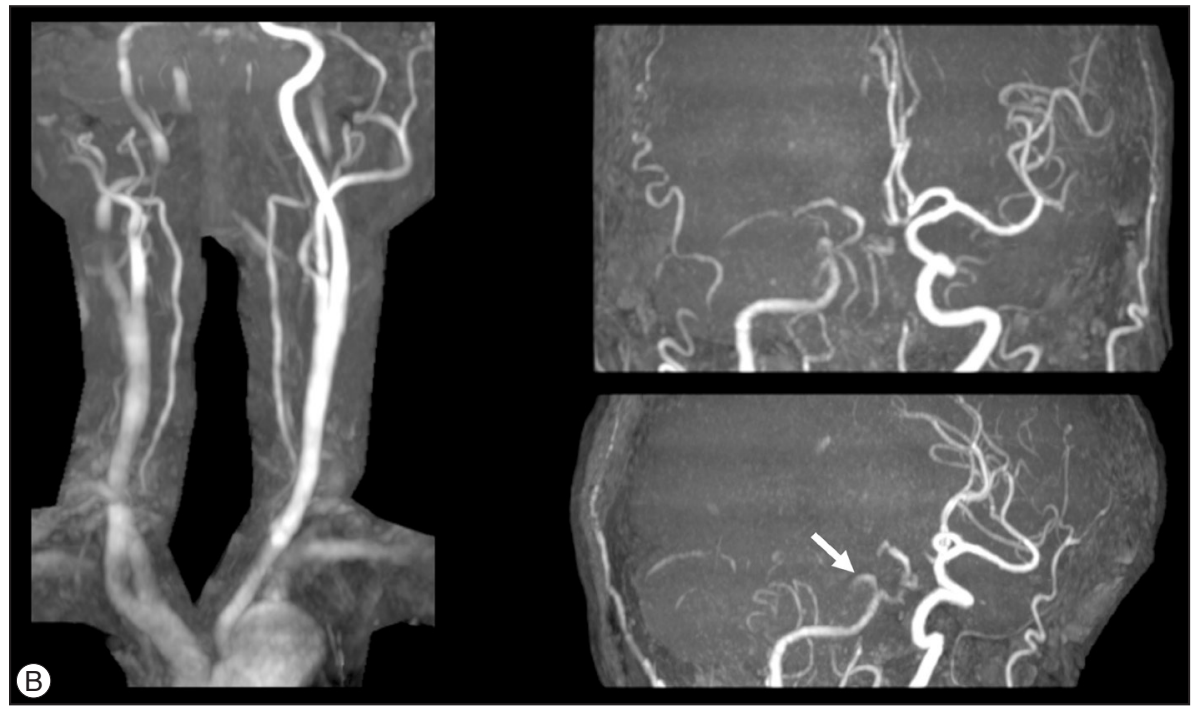

sciousness and was transported to our hospital 40 minutes after symptom onset. On admission, he was comatose with a Glasgow Coma Scale score of 6 and quadriplegic, with pupillary mydriasis and absence of corneal reflexes bilaterally. The National Institutes of Health Stroke Scale score was 38. Magnetic resonance diffusion-weighted imaging (DWI) showed an extensive acute infarction in the right cerebral hemisphere (Fig. 1A). Magnetic resonance angiography (MRA) showed occlusion of the right middle cerebral artery (MCA) and BA (Fig. 1B). The extra-cranial vertebral arteries (VAs) were comparatively well visualized on MRA, but the intracranial VAs and the BA were poorly visualized (Fig. 1B). Because electrocardiogram on admission showed atrial fibrillation, multiple acute infarctions due to cardiogenic emboli were suspected. Early ischemic changes in the territory of the right MCA were presented in more than one third of the MCA territory with a DWI Alberta Stroke Programme Early Computed Tomographic (CT) Score of 3; therefore, the use of intravenous tissue plasminogen activator was contraindicated because of the high risk of hemorrhage and endovascular revascularization of the anterior circulation was not indicated. However, the patient's severely impaired level of consciousness was thought to be caused by the BA occlusion and the infarction in the territory of the BA excluding the right occipital lobe was small. Accordingly, we attempted to rescue the brainstem by endovascular recanalization of the BA.

Diagnostic angiography showed reduced opacification in the segments from bilateral VAs to mid-basilar artery (Fig. 2A). Carotid angiography revealed the presence of a PPTA arising from the precavernous portion of the right internal carotid artery (ICA) (Fig. 2B, C). This finding was consistent with preoperative MRA that showed unusual branching from the precavernous portion of the right ICA (Fig. 1B). Because the sizes of bilateral VAs were relatively small, we choose the anterior-toposterior circulation approach for thrombectomy.

We selected the PPTA as the access route for the BA, and an 8 Fr Merci Balloon Guiding Catheter (Concentric Medical, Mountain View, CA, USA) was inserted into the origin of the right ICA. A Merci Microcatheter (Concentric Medical, Mountain View, CA, USA) was introduced into the BA through the PPTA, and the BA occlusion was crossed with the microcatheter. Simultaneous angiography from the guiding catheter and the microcatheter indicated the location of the clot (Fig. 2D). Because the infarction in the territory of the right posterior cerebral artery (PCA) was extensive, we chose the left PCA for positioning the microcatheter distal to the clot. A Merci Retriever V 2.5 Soft (Concentric Medical, Mountain View, CA, USA) was inserted into the left PCA and retrieval was performed slowly (Fig. 2E). However, the clot was not retrieved and the occlusion was not recanalized. Although we also used a Merci Retriever V 2.0 Firm (Concentric Medical, Mountain View, CA, USA), the result was the same. Because we thought the clot was hard, we positioned the microcatheter at the origin of the occlusion and performed local intraarterial thrombolysis with 12000 IU of urokinase with the intention of softening the clot. We then used the Merci Retriever V 2.0 Firm again for mechanical thrombectomy and the BA occlusion was success- 
fully recanalized. A small amount of the clot was retrieved, but the distal arteries of the left PCA were occluded by thrombus. Therefore, we added an intraarterial infusion of 12000 IU of urokinase into the left PCA. Although the distal arteries of the PCA were not completely recanalized, we terminated the intraarterial thrombolysis to avoid urokinase-induced hemorrhage. The final angiography showed relatively successful revascularization of the BA occlusion with a Thrombolysis in Cerebral In- farction (TICI) score of 2B (Fig. 2F, G). Brain CT after the endovascular treatment showed no intracranial hemorrhage. Magnetic resonance imaging at 24 hours after treatment showed relatively small, new infarctions in the left occipital lobe, left thalamus, and midbrain compared with DWI on admission without marked extension of infarctions in the right MCA area (Fig. 3A). MRA showed good patency of the BA after endovascular revascularization (Fig. 3B). The infarction in the territory
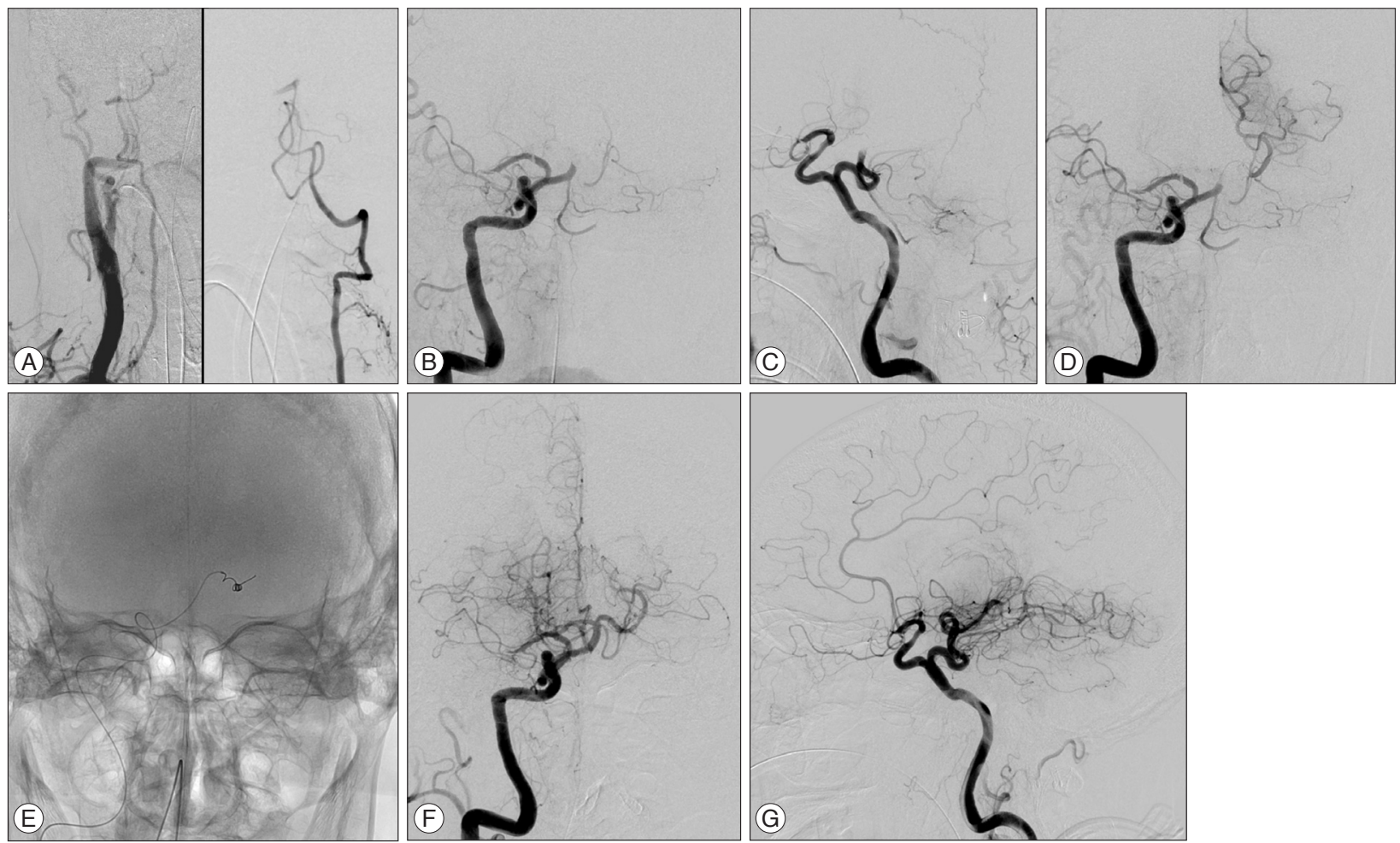

Fig. 2. A : Right innominate angiography showing a relatively hypoplastic right VA (left). Left vertebral angiography showing occlusion of the BA (right). The intracranial left VA is relatively hypoplastic. B and C : Right carotid angiography showing the occluded right middle cerebral artery and a persistent primitive trigeminal artery arising from the precavernous portion of the right internal carotid artery. D : Simultaneous angiography from the guiding catheter and the microcatheter indicating the location of the clot. $\mathrm{E}$ : Radioscopic image showing the Merci Retriever inserted into the left posterior cerebral artery. F and G : Final angiography after the endovascular treatment showing recanalization of the BA. VA : vertebral artery, BA : basilar artery.
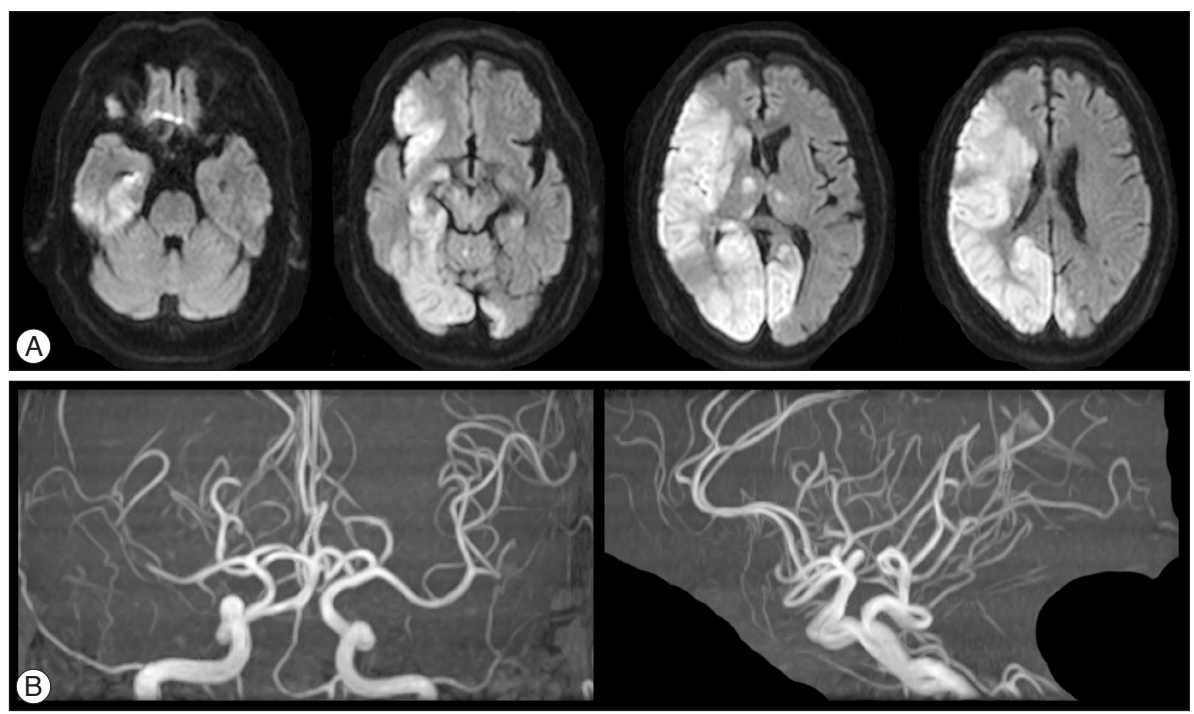

Fig. 3. A : Magnetic resonance DWI at 24 hours after treatment showing new, relatively small infarctions in the left occipital lobe, left thalamus, and midbrain compared with DWI on admission. B : MRA after treatment showing good patency of the BA after the endovascular revascularization. DWI : diffusion-weighted imaging, BA : basilar artery. 
of the right MCA did not extend thereafter and did not require any surgical procedure such as external craniectomy.

After treatment, the patient's consciousness and right motor weakness improved. At 90 days after treatment, although his left hemiparesis was moderate, he was lucid and able to eat using his right hand, with a modified Rankin Scale score of 3.

\section{DISCUSSION}

The present case raises some important clinical issues. First, although persistent carotid-vertebrobasilar anastomoses such as a PPTA are relatively rare vascular anomalies, the possibility of their presence should be considered when diagnosing and treating a patient with acute ischemic stroke. Second, for endovascular revascularization in cases of acute BA occlusion, persistent carotid-vertebrobasilar anastomoses are good access options when available, as was shown in our patient. Finally, the present case is a rare case of major artery occlusions in both the anterior and posterior circulation occurring simultaneously.

A PPTA is a relatively rare vascular anomaly, but is the most common carotid-vertebrobasilar anastomosis, with a reported incidence of $0.03-2.2 \%{ }^{22)}$. It usually arises from the cavernous or precavernous portion of the ICA and terminates at the BA between the origin of the superior cerebellar artery and anterior inferior cerebellar artery. Among the reported cases of cerebral ischemia associated with a PPTA ${ }^{1-5,7,9,10,17,18,20,21,23)}$, most involved relatively small infarctions in the territory of the BA because of artery-to-artery embolism of plaques in cervical carotid artery stenosis or dissection. Only a few cases of major stroke, such as an acute BA occlusion, have been reported in the literature ${ }^{7)}$. However, major ischemic stroke with an unusual pattern of involved territories may be associated with these cerebral vascular anomalies. In the present case, the infarctions involved both the anterior and posterior circulation, and this was suspected to be due to the same cardiogenic embolus passing through the ipsilateral ICA.

There are few reports of endovascular treatment for cerebral ischemia associated with a PPTA. Fuse et al. ${ }^{2)}$ reported a case of acute occlusion in the terminal portion of an ICA associated with a PPTA that was treated by local intraarterial fibrinolysis using urokinase in the ICA. Ito et al. ${ }^{7)}$ reported a case of acute BA occlusion associated with a PPTA that was treated by local intraarterial fibrinolysis using urokinase, but the VA in that case was not hypoplastic and the access route for the BA was not mentioned. In the cases of other vascular diseases closely associated with a PPTA such as aneurysm or arteriovenous fistula involving the PPTA, endovascular treatment using the PPTA as the target vessel was well described ${ }^{8)}$.

Concerning the anterior-to-posterior circulation approach used in the present case, there are few reports of endovascular revascularization for acute ischemic stroke. Liu et al. ${ }^{14)}$ reported a case of endovascular revascularization for acute BA occlusion via a posterior communicating artery. Although there are two reported cases of coil embolization of a posterior circulation aneurysm via a PPTA ${ }^{6,19)}$, to our knowledge, the present case is the first report of endovascular treatment for acute BA occlusion via a PPTA. Because the PPTA was larger than the VAs in the present case, we chose the PPTA as the access route for the BA. We used a Merci Retriever because it was the only approved device for mechanical thrombectomy at that time. However, revascularization was not achieved after two passes using two types of Merci Retriever. The clot was thought to be hard because we felt substantial resistance on traversing the occlusion with the microcatheter. It has been reported that combined mechanical and intraarterial pharmacological therapy was associated with higher recanalization rates than either intervention alone, and softening the clot by thrombolytic agent was considered to be one of the mechanisms ${ }^{11}$. In addition, it has been reported that multiple number of Merci retrieval attempts increased the risk of complications such as subarachnoid hemorrhage ${ }^{15)}$. Therefore, we used adjunctive intraarterial thrombolysis with urokinase for softening the clot before further retrial of mechanical thrombectomy. We started with urokinase of 12000 IU according to the method of the MCA embolism local fibrinolytic intervention trial (MELT) ${ }^{16)}$, and successful revascularization was achieved with the additional use of the Merci Retriever. However, the possibility of vascular damage must be considered because the PPTA is a relatively tortuous vessel. No hemorrhage was detected in our case, but patients should be monitored carefully for hemorrhagic complications such as subarachnoid hemorrhage. In the present case, the TICI score was $2 \mathrm{~B}$ and new infarction in the territory of the left PCA was observed in the postoperative magnetic resonance imaging. Other devices such as large bore aspiration catheter and stent retriever, which might be safer for vascular damage and more effective for revascularization than the Merci Retriever, could also be used via the PPTA.

The present case is a rare case of major artery occlusions in both the anterior and posterior circulation occurring simultaneously. Many published case series have demonstrated that the time window for revascularization of the posterior circulation is longer than that of the anterior circulation, and it has been speculated that the territory of the BA has different collateral flow patterns and lower hemorrhage rates compared with the territory of the ICA ${ }^{12,13)}$. The present case supports the concept of a longer treatable window for the posterior circulation. Therefore, revascularization of the posterior circulation should be considered separately from that for the anterior circulation, when infarctions involve both the anterior and posterior circulation. Clinicians should be aware that the persistent primitive artery can be associated with acute ischemic stroke involving both the anterior and posterior circulation simultaneously. In this rare situation, even in patients with a major infarction in the anterior circulation, there might still be an opportunity for mechanical thrombectomy especially for a lesion in the posterior circulation, which has a longer time window for treatment 
than that of the anterior circulation.

\section{CONCLUSION}

Although persistent carotid-vertebrobasilar anastomoses such as a PPTA are relatively rare vascular anomalies, if the persistent primitive artery is present, it can be an access route for mechanical thrombectomy for acute ischemic stroke. Clinicians should be aware that the persistent primitive artery can be associated with acute ischemic stroke involving both the anterior and posterior circulation simultaneously. In this rare situation, even in patients with a major infarction in the anterior circulation, there might still be an opportunity for mechanical thrombectomy especially for a lesion in the posterior circulation, which has longer time window for treatment than that of the anterior circulation.

\section{References}

1. Foerch C, Berkefeld J, Halbsguth A, Ziemann U, Neumann-Haefelin T : Brain stem infarction caused by proximal internal carotid artery stenosis in a patient with a persisting primitive trigeminal artery. Cerebrovasc Dis 22 : 200-202, 2006

2. Fuse T, Niwa Y, Harada $S$ : Local intraarterial fibrinolytic therapy for embolic stroke associated with vascular anomalies--two case reports. Neurol Med Chir (Tokyo) 40 : 641-644, 2000

3. Gasecki AP, Fox AJ, Lebrun LH, Daneault N : Bilateral occipital infarctions associated with carotid stenosis in a patient with persistent trigeminal artery. The Collaborators of the North American Carotid Endarterectomy Trial (NASCET). Stroke 25 : 1520-1523, 1994

4. Gaughen JR, Starke RM, Durst CR, Evans AJ, Jensen ME : Persistent trigeminal artery : in situ thrombosis and associated perforating vessel infarction. J Clin Neurosci 21 : 1075-1077, 2014

5. Iancu D, Anxionnat R, Bracard S : Brainstem infarction in a patient with internal carotid dissection and persistent trigeminal artery : a case report. BMC Med Imaging $10: 14,2010$

6. Ikushima I, Arikawa S, Korogi Y, Uehara H, Komohara Y, Takahashi M : Basilar artery aneurysm treated with coil embolization via persistent primitive trigeminal artery. Cardiovasc Intervent Radiol 25 : 70-71, 2002

7. Ito $\mathrm{Y}$, Watanabe H, Niwa H, Hakusui S, Ando T, Yasuda T, et al. : The protective effect of a persistent trigeminal artery on brain stem infarctions : a follow-up case report. Intern Med 37 : 334-337, 1998

8. Jin SC, Park H, Kwon do H, Choi CG : Direct carotid cavernous fistula of an adult-type persistent primitive trigeminal artery with multiple vascular variations. J Korean Neurosurg Soc 49 : 226-228, 2011
9. Khodadad G : Trigeminal artery and occlusive cerebrovascular disease. Stroke 8 : 177-181, 1977

10. Lewis VL, Cail WS : Persistent trigeminal artery with internal carotid artery occlusion. Neurosurgery $13: 314-315,1983$

11. Lin R, Vora N, Zaidi S, Aleu A, Jankowitz B, Thomas A, et al. : Mechanical approaches combined with intra-arterial pharmacological therapy are associated with higher recanalization rates than either intervention alone in revascularization of acute carotid terminus occlusion. Stroke 40 : 2092-2097, 2009

12. Lindsberg PJ, Mattle HP : Therapy of basilar artery occlusion : a systematic analysis comparing intra-arterial and intravenous thrombolysis. Stroke 37 : 922-928, 2006

13. Lindsberg PJ, Sairanen T, Strbian D, Kaste M : Current treatment of basilar artery occlusion. Ann N Y Acad Sci 1268 : 35-44, 2012

14. Liu W, Kung DK, Mahaney KB, Rossen JD, Jabbour PM, Hasan DM : Anterior-to-posterior circulation approach for mechanical thrombectomy of an acutely occluded basilar artery using the penumbra aspiration system. World Neurosurg 77 : 398.E17-398.E20, 2012

15. Loh Y, Jahan R, McArthur DL, Shi ZS, Gonzalez NR, Duckwiler GR, et al. : Recanalization rates decrease with increasing thrombectomy attempts. AJNR Am J Neuroradiol 31 : 935-939, 2010

16. Ogawa A, Mori E, Minematsu K, Taki W, Takahashi A, Nemoto S, et al. : Randomized trial of intraarterial infusion of urokinase within 6 hours of middle cerebral artery stroke : the middle cerebral artery embolism local fibrinolytic intervention trial (MELT) Japan. Stroke 38 : 26332639, 2007

17. Okada Y, Shima T, Nishida M, Yamada T, Yamane K, Okita S, et al. : Bilateral persistent trigeminal arteries presenting with brain-stem infarction. Neuroradiology $34: 283-286,1992$

18. Sannegowda RB, Srivastava T, Jain RS, Mathur T, Jain R : Brainstem transient ischemic attacks due to compression of pons from a persistent primitive trigeminal artery. Neurol India $61: 321-322,2013$

19. Schlamann M, Doerfler A, Schoch B, Forsting M, Wanke I : Balloon-assisted coil embolization of a posterior cerebral artery aneurysm via a persistent primitive trigeminal artery : technical note. Neuroradiology 48: 931-934, 2006

20. Schwartz NE, Albers GW : Acute strokes in the setting of a persistent primitive trigeminal artery. BMJ Case Rep 2009 : bcr2006111773, 2009

21. Suzuki S, Chang GY : A case of primitive trigeminal artery infarction. Neurology 75 (18 Suppl 1) : S66, 2010

22. Vasović L, Jovanović I, Ugrenović S, Vlajković S, Jovanović P, Stojanović $\mathrm{V}$ : Trigeminal artery : a review of normal and pathological features. Childs Nerv Syst $28: 33-46,2012$

23. Waller FT, Simons RL, Kerber C, Kiesel IO, Tanabe CT : Trigeminal artery and microemboli to the brain stem. Report of two cases. J Neurosurg 46 : 104-106, 1977 